\title{
LOCALLY RISK-MINIMIZING HEDGING OF INSURANCE PAYMENT STREAMS
}

BY

\author{
MARTIN RIESNER
}

\begin{abstract}
For the martingale case Föllmer and Sondermann (1986) introduced a unique admissible risk-minimizing hedging strategy for any square-integrable contingent claim $H$. Schweizer (1991) developed their theory further to the semimartingale case introducing the notion of local risk-minimization. Møller (2001) extended the theory of Föllmer and Sondermann (1986) to hedge general payment processes occurring mainly in insurance. We expand local risk-minimization to the theory of hedging general payment processes and derive such a hedging strategy for general unit-linked life insurance contracts in a general Lévy process financial market.
\end{abstract}

\section{KEYWORDS}

Local risk-minimization, semimartingales, hedging, incomplete market, Lévy process, unit-linked life insurance, Markov chain.

\section{INTRODUCTION}

In this paper we consider life insurance contracts driven by a Markov jump process and having unit-linked benefits (and possibly premiums) based on the Lévy-process stock model of Chan (1999). Those benefits are usually due immediately upon occurrence of some insurance event at random times. In life insurance such an event could be for instance a disability or the death of the policy-holder. Similarly, premiums are paid according to a predefined premium scheme for a fixed time or as long as the policy-holder is alive. For those general contracts we derive a locally risk-minimizing hedging strategy and compute additionally the involved hedging risk, which has a local interpretation under the historical measure. This is an important figure, since, as in reality, we face an incomplete financial market in which riskless hedging is not possible. The model and the results presented here complement Møller (2001) who derives risk-minimizing hedging strategies for unit-linked life insurance payment streams in the same multi-state Markov model but for a Black-Scholes martingale 
financial market. Moreover, in analogy to Riesner (2006a) we show for the generalized model that the involved hedging risk can be separated in two components: financial risk and insurance risk. In Møller (2001) the pure financial risk is not present, since for a complete financial market, turned incomplete by the mortality law, only the insurance risk appears.

To obtain a hedging strategy for a square-integrable contingent claim with fixed maturity in a semimartingale financial market Schweizer (1991) developed the theory of local risk-minimization. This theory is an extension of risk-minimization developed earlier by Föllmer and Sondermann (1986) for the martingale case. Møller (2001) enlarged the theory of Föllmer and Sondermann (1986) to risk-minimizing hedging of general payoff streams, where the hedgers' liabilities are given by a square-integrable payment process. In the Appendix we justify this concept to be embedded without any difficulties in the theory of local risk-minimization (for the semimartingale case). We are therefore allowed to apply it to our setting.

Additionally, we would like to mention Møller (1998) who began the analysis of such hedging strategies in a Black-Scholes financial market. To apply the local risk-minimizing hedging theory for square-integrable contingent claims with fixed maturity Møller (1998) restricts himself to insurance contracts having a single premium payment at the beginning and paying benefits at the end of the considered time horizon only. This has also been generalized in Riesner (2006a) towards a general Lévy process financial market.

\section{THE MODEL}

We consider a filtered probability space $\left(\Omega, \mathbb{F},\left(\mathcal{F}_{t}\right)_{0 \leq t \leq T}, \mathbb{P}\right)$ modeled as a product space of two independent filtered probability spaces: $\left(\Omega_{1}, \mathbb{G},\left(\mathcal{G}_{t}\right)_{0 \leq t \leq T}, \mathbb{P}_{1}\right)$ describing the financial market and $\left(\Omega_{2}, \mathbb{U},\left(\mathcal{H}_{t}\right)_{0 \leq t \leq T}, \mathbb{P}_{2}\right)$ describing the insurance portfolio. The filtrations $\left(\mathcal{G}_{t}\right)_{0 \leq t \leq T}$ and $\left(\mathcal{H}_{t}\right)_{0 \leq t \leq T}$ are used to construct the filtration $\left(\mathcal{F}_{t}\right)_{0 \leq t \leq T}$ via $\mathcal{F}_{t}=\mathcal{G}_{t} \otimes \mathcal{H}_{t}$, each $0 \leq t \leq T$. All mentioned probability spaces are assumed to satisfy the usual hypothesis of right continuity and completeness. $T>0$ is the finite time-horizon. $\mathcal{P}$ and $O$ denote the predictable and the optional $\sigma$-algebra on $\Omega \times[0, T]$, respectively.

\section{Financial market}

The Lévy-process financial market model that we will work with is introduced in Chan (1999). Given a (càdlàg) Lévy process $L=\left(L_{t}\right)_{0 \leq t \leq T}$ with $L_{0}=0$ a.s., it consists of a risky investment $S=\left(S_{t}\right)_{0 \leq t \leq T}$ and a risk-free investment alternative $B=\left(B_{t}\right)_{0 \leq t \leq T}$ given by

$$
\begin{gathered}
d S_{t}=S_{t-}\left(b_{t} d t+\sigma_{t} d L_{t}\right), S_{0}>0, \\
d B_{t}=r_{t} B_{t} d t, B_{0}=1,
\end{gathered}
$$


where the drift $b_{t}$, the volatility $\sigma_{t}>0$ and the risk-free interest rate $r_{t}$ are supposed to be continuous and deterministic functions on $[0, T]$. The coefficient functions $f(t, x)=b_{t} x$ and $g(t, x)=\sigma_{t} x$ are obviously process-Lipschitz. Therefore the solution of (1) is unique (cf. Protter (2004), Chapter V, Section 3, Theorem 6 and 7). The filtration $\mathcal{G}_{t}$ is supposed to be the completed $\sigma$-algebra $\sigma\left(L_{s}, 0 \leq s \leq t\right)$. We denote by $N(d t, d x)$ the Poisson random measure on $[0, \infty) \times$ $\mathbb{R} \backslash\{0\}$ corresponding to the jumps of $L$ and given the Lévy measure $v(d x)$ of $L$ we define the corresponding compensated measure by $M(d t, d x):=N(d t, d x)-$ $d t v(d x)$ ( $d t$ denotes the Lebesgue measure). For notational convenience we set $N_{t}(\{0\}) \equiv v(\{0\})=0$ and $\Delta f(t)=f(t)-f(t-)$ for any suitable function $f$. The Lévy measure $v(d x)$ satisfies in general $\int_{\mathbb{R}} \min \left(1, x^{2}\right) v(d x)<\infty$. We assume further that

$$
\int_{\{|x|>1\}}|x|^{3} v(d x)<\infty
$$

Assumption (2) (cf. Chan (1999)) allows one to decompose the process $L$ into

$$
L_{t}=c W_{t}+M_{t}+a t,
$$

where $\left(c W_{t}\right)_{(0 \leq t \leq T)}$ is a Brownian Motion with variance $c^{2} t$ for some $c \in(0, \infty)$ and $M_{t}=\int_{0}^{t} \int_{\mathbb{R}} x M(d s, d x), 0 \leq t \leq T$, is a square-integrable martingale. Finally, $a=\mathbb{E}\left(L_{1}\right)$. To have $S_{t}>0$, we assume $\Delta L_{t}=\Delta M_{t}>-1 / \sigma_{t} \mathbb{P}_{1}$-a.s. and all $0 \leq t \leq T$. The discounted stock price $\hat{S}_{t}=S_{t} B_{t}^{-1}$ is square-integrable and admits the following semimartingale decomposition:

$$
\hat{S}_{t}=S_{0}+\tilde{M}_{t}+\tilde{A}_{t},
$$

where $\tilde{M}_{t}=\int_{0}^{t} \sigma_{s} \hat{S}_{s-} d\left(c W_{s}+M_{s}\right)$ is a $\mathbb{P}_{1}$-martingale and $\tilde{A}_{t}=\int_{0}^{t}\left(a \sigma_{s}+b_{s}-r_{s}\right) \hat{S}_{s-}$ $d s$ is a continuous, adapted and hence predictable process. There exist arbitrary many measures equivalent to $\mathbb{P}_{1}$ such that $\hat{S}$ is a martingale under such a measure (cf. Chan (1999)). One such measure is the Föllmer-Schweizer measure which we denote by $\mathbb{Q}_{1}$ and which we choose to find a locally risk-minimizing hedging strategy (cf. Appendix A.1). Its construction is described in Schweizer (1991), Chan (1999) and is repeated in Riesner (2006a). With this measure we consider in the following the risk-neutral financial market $\left(\Omega_{1}, \mathbb{G},\left(\mathcal{G}_{t}\right)_{0 \leq t \leq T}, \mathbb{Q}_{1}\right)$. $M^{\mathbb{Q}}(d s, d x)$ denotes the compensated jump measure of $L$ under $\mathbb{Q}_{1}$. Its compensator is given by

$$
v_{t}^{\mathbb{Q}}(d x) d t=\left(1+G_{t} x\right) v(d x) d t
$$

with Girsanov parameter $G_{t}=\frac{r_{t}-b_{t}-a \sigma_{t}}{\sigma_{t}\left(c^{2}+v\right)}$ and $v=\int_{\mathbb{R}} x^{2} v(d x) . \hat{S}$ is a square-integrable martingale under $\mathbb{Q}_{1}$ and admits the decomposition

$$
d \hat{S}_{t}=\sigma_{t} \hat{S}_{t-} d\left(c W_{t}^{\mathbb{Q}}+M_{t}^{\mathbb{Q}}\right),
$$


where $W^{\mathbb{Q}}$ is a standard Brownian motion under $\mathbb{Q}_{1}$ and $M_{t}^{\mathbb{Q}}=\int_{0}^{l} \int_{\mathbb{R}} x M^{\mathbb{Q}}(d s, d x)$, $0 \leq t \leq T$, is the compensated jump process of $L$ under $\mathbb{Q}_{1}$. Note that because of the additional assumption (2) $v$ and $v^{\mathbb{Q}}(\cdot)$ are well-defined and that moreover $M_{t}^{\mathbb{Q}}$ is square-integrable.

\section{Life insurance}

One possible model to describe $\left(\Omega_{2}, \mathbb{H},\left(\mathcal{H}_{t}\right)_{0 \leq t \leq T}, \mathbb{P}_{2}\right)$ is the classical multi-state Markov model of Hoem (1969); see also Møller (2001). One considers the set $\mathcal{I}=\{0,1, \ldots, J\}$ of possible states of a policy where usually one assumes 0 to be its initial state. $\mathcal{I}=$ active, disabled, dead $\}$ describes exemplarily three possible states of an insured individual. A càdlàg Markov process $Z=\left(Z_{t}\right)_{0 \leq t \leq T}$ with values in $\mathcal{I}$ and initial distribution $(1,0, \ldots, 0)$ is now used to indicate the state of the policy at time $t$. One further defines $\mathcal{H}_{t}$ to be the completed $\sigma$-algebra $\sigma\left(Z_{s}, 0 \leq s \leq t\right)$. In order to count the number of transitions from state $j$ to state $k$ in the time interval $(0, t]$ a multivariate counting process $\left(N^{j k}\right)_{j \neq k}$ is defined by

$$
N_{t}^{j k}=\#\left\{s \mid s \in(0, t], Z_{s-}=j, Z_{s}=k\right\} \quad j, k \in \mathcal{J}, \quad j \neq k, 0 \leq t \leq T .
$$

Moreover the processes $I_{t}^{j}=\mathbb{1}_{\left\{Z_{t}=j\right\}}, j \in \mathcal{I}$, are introduced indicating whether the policy is in state $j$ at time $t$ or not. The Markov chain $Z$ is further assumed to posses transition rates $\lambda^{j k}$ given by

$$
\lambda_{t}^{j k}=I_{t-}^{j} \mu_{t}^{j k}, \quad 0 \leq t \leq T
$$

where the intensities of transition $\mu_{t}^{j k}$ are supposed to exist and to be deterministic, continuous functions. The transition rates $\lambda^{j k}$ compensate the counting processes $N^{j k}$ resulting in square-integrable, mutually orthogonal and zero-mean martingales

$$
M_{t}^{j k}=N_{t}^{j k}-\int_{0}^{t} \lambda_{u}^{j k} d u, \quad 0 \leq t \leq T
$$

The orthogonality follows from the fact that the $N^{j k}$ do not have any simultaneous jumps (cf. Kallenberg (2002), Lemma 15.6). The predictable quadratic variation process $\left\langle M^{j k}, M^{j k}\right\rangle_{t}$ is hence given by

$$
\left\langle M^{j k}, M^{j k}\right\rangle_{t}=\int_{0}^{t} \lambda_{u}^{j k} d u=\int_{0}^{t} I_{u}^{j} \mu_{u}^{j k} d u, \quad 0 \leq t \leq T .
$$

Given the intensities of transition the transition probabilities $p_{j k}(t, u)=\mathbb{P}_{2}\left(Z_{u}=\right.$ $k \mid Z_{t}=j$ ) of $Z$ are determined by Kolmogorov's backward differential equations

$$
\frac{d}{d t} p_{j k}(t, u)=\sum_{l: l \neq j} \mu_{t}^{j l}\left(p_{j k}(t, u)-p_{l k}(t, u)\right), \quad 0 \leq t \leq u \leq T,
$$


subject to the conditions $p_{j k}(u, u)=\mathbb{1}_{\{j=k\}}$. We follow Aase and Persson (1994) and Møller $(1998,2001)$ in assuming risk-neutrality of an insurance company towards mortality.

\section{LOCALLY RISK-MINIMIZING HEDGING}

Using the independence of the financial market and the insurance portfolio we work in the following on the product space $(\Omega, \mathbb{E}, \mathbb{Q})$ where $\mathbb{Q}$ denotes the product measure of $\mathbb{Q}_{1}$ and $\mathbb{P}_{2}$. This is the risk-neutral measure for the insurer facing the hedging problem of unit-linked life insurance contracts. The multi-state Markov insurance model admits quite general forms of benefit and premium payments (cf. Møller (2001)). First, it might be the case that a transition from state $j$ to state $k$ at time $t$ immediately induces a payment $g_{t}^{j k}=g^{j k}\left(t, S_{t}\right)$ and second, it is possible that depending on the policy sojourning in state $j$ the insurance company continuously pays the rate $g_{t}^{j}=g^{j}\left(t, S_{t}\right)$ at time $t$. Payments of the first type usually occur with general life insurances whereas state-wise life annuities typically generate payments of the second type. Being in state $j$, the policy shall additionally admit lump-sum annuity payments $\tilde{g}_{t}^{j}=\tilde{g}^{j}\left(t, S_{t}\right)$ at fixed deterministic times $t \in \Gamma=\left\{\tau_{1}, \ldots, \tau_{n}\right\}$ for some $n \geq 1$. The amount payable at time $t \in[0, T]$, depending on the policy being in state $j$, is therefore equal to $G_{t}^{j}=G^{j}\left(t, S_{t}\right)=g_{t}^{j}+\tilde{g}_{t}^{j} 1_{\{t \in \Gamma\}}$ and up to time $t$ the total state-wise annuity payment is equal to $\int_{0}^{p} G^{j}\left(u, S_{u}\right) d u=\int_{0}^{t} g^{j}\left(u, S_{u}\right) d u+\sum_{\tau \in \Gamma, \tau \leq t} \tilde{g}^{j}\left(\tau, S_{\tau}\right)$. Here and for the rest of this paper we treat $\mathbb{1}_{\{u \in \Gamma\}} d u$ as discrete counting measure on the set $\Gamma$ with jump hight one. This enables us to discuss the continuous and the discrete case simultaneously. Moreover, we assume benefit and premium payments represented as difference in the functions $g^{j k}, g^{j}$ and $\tilde{g}^{j}$, where negative values are premium payments. For technical reasons $(t, s) \mapsto g^{j k}(t, s),(t, s) \mapsto$ $g^{j}(t, s)$ and $(t, s) \mapsto \tilde{g}^{j}(t, s) \mathbb{1}_{\{t \in \Gamma\}}$ are supposed to be measurable for $j, k \in \mathcal{I}$, and we need that

$$
\sup _{u \in[0, T]} \mathbb{E}^{\mathbb{Q}}\left[\left(B_{u}^{-1} g\left(u, S_{u}\right)\right)^{2}\right]<\infty, \text { for all } g^{j k}, g^{j}, \tilde{g}^{j} 1_{\{\cdot \in \Gamma\}}
$$

That condition guarantees that each well-defined stochastic integral of $B_{u}^{-1} g\left(u, S_{u}\right)$ with respect to a square-integrable martingale is a square-integrable martingale. Next we consider the arbitrage-free price process of those insurance claims given for $0 \leq t \leq u \leq T$ by

$$
\begin{aligned}
F^{j k}\left(t, S_{t}, u\right) & :=\mathbb{E}^{\mathbb{Q}}\left[B_{t} B_{u}^{-1} g^{j k}\left(u, S_{u}\right) \mid \mathcal{G}_{t}\right], \\
F^{j}\left(t, S_{t}, u\right) & :=\mathbb{E}^{\mathbb{Q}}\left[B_{t} B_{u}^{-1} G^{j}\left(u, S_{u}\right) \mid \mathcal{G}_{t}\right] .
\end{aligned}
$$

Obviously we have $F^{j}\left(t, S_{t}, u\right)=\mathbb{E}^{\mathbb{Q}}\left[B_{t} B_{u}^{-1} g_{u}^{j} \mid \mathcal{G}_{t}\right]+\mathbb{E}^{\mathbb{Q}}\left[B_{t} B_{u}^{-1} \tilde{g}_{u}^{j} 1_{\{u \in \Gamma\}} \mid \mathcal{G}_{t}\right]$. The independence of the financial market and the insurance portfolio allows us 
to condition the expectations above only with the information described by $\mathcal{G}_{t}$. $F \cdot\left(t, S_{t}, u\right), 0 \leq t \leq u \leq T$, is the price at time $t$ of an insurance claim due at time $u$. The superscript "." either stands for the state $j$ or for a transition $j k$ from state $j$ to state $k$. In order to perform the following calculations properly we suppose that the functions $F^{\cdot}(\cdot, \cdot, u) \in C^{1,2}([0, T] \times[0, \infty))$ for each $u \in[0, T]$, that is, once continuously differentiable in the first and twice continuously differentiable in the second variable. For notational convenience we denote by $F^{\cdot}(t, x, u)=\frac{d}{d x} F^{\cdot}(t, x, u)$. A further assumption is that $F^{\cdot \prime}(t, x, u)$ is uniformly bounded, i.e. there exists a non-random constant $c_{1}>0$ such that

$$
\left|F^{\cdot}(t, x, u)\right| \leq c_{1}<\infty \forall t, x, u \text { and for all } F^{j}, F^{j k} \mathbb{Q} \text {-a.s. }
$$

Later we need the Galtchouk-Kunita-Watanabe decomposition (cf. (12)) of the discounted price processes $B_{t}^{-1} F \cdot\left(t, S_{t}, u\right)$.

Proposition 3.1. Let $v_{t}^{\mathbb{Q}}=\int_{\mathbb{R}} x^{2} v_{t}^{\mathbb{Q}}(d x)$ and $\kappa_{t}=\left(c^{2}+v_{t}^{\mathbb{Q}}\right), t \in[0, T]$. For any price process $F$ and $0 \leq t \leq u \leq T$ the Galtchouk-Kunita-Watanabe decomposition of $B_{t}^{-1} F \cdot\left(t, S_{t}, u\right)$ is given by

$$
B_{t}^{-1} F \cdot\left(t, S_{t}, u\right)=F \cdot\left(0, S_{0}, u\right)+\int_{0}^{t} \xi_{\tau}(u) d \hat{S}_{\tau}+K_{t}(u),
$$

where

$$
\xi_{\tau}(u)=\frac{c^{2}}{\kappa_{t}} F^{\cdot \prime}\left(t, S_{t-}, u\right)+\frac{1}{\sigma_{t} \hat{S}_{t-} \kappa_{t}} \int_{\mathbb{R}} x J \cdot(t, x, u) v_{t}^{\mathbb{Q}}(d x)
$$

with

$$
J \cdot(t, x, u)=B_{t}^{-1}\left\{F \cdot\left(t, S_{t-}+\sigma_{t} S_{t-} x, u\right)-F \cdot\left(t, S_{t-}, u\right)\right\},
$$

and

$$
K_{t}(u)=\int_{0}^{t} \zeta_{\tau}^{(1) \cdot}(u) d W_{\tau}^{\mathbb{Q}}+\int_{0}^{t} \int_{\mathbb{R}^{\prime}} \zeta_{\tau}^{(2)} \cdot(y, u) M^{\mathbb{Q}}(d \tau, d y)
$$

is orthogonal to $\hat{S}$. Further the processes $\zeta_{t}^{(1)}(u)$ and $\zeta_{t}^{(2)}(y, u)$ are given by

$$
\begin{aligned}
\zeta_{t}^{(1)}(u) & =c \sigma_{t} \hat{S}_{t-}\left(F^{\prime}\left(t, S_{t-}, u\right)-\xi_{t}(u)\right), \\
\zeta_{t}^{(2)}(y, u) & =J(t, y, u)-\sigma_{t} \hat{S}_{t-} \xi_{t}(u) y .
\end{aligned}
$$

Proof. We refer to Appendix A of Riesner (2006a) for an explanation of the decomposition. Additionally we show here that the integrals with respect to $\hat{S}$, $W^{\mathbb{Q}}$ and $M^{\mathbb{Q}}(d t, d y)$ are really square-integrable. Since it is continuous in $t$ and (2) holds we have that

$$
\sup _{t \in[0, T]}\left|\int_{\mathbb{R}} x^{2} v_{t}^{\mathbb{Q}}(d x)\right| \leq \int_{\mathbb{R}} x^{2} v(d x)+\sup _{t \in[0, T]}\left|G_{t}\right|\left|\int_{\mathbb{R}} x^{3} v(d x)\right|<\infty .
$$


The mean-value theorem yields $J(t, x, u)=F^{\prime}\left(t, x_{0}, u\right) \sigma_{t} \hat{S}_{t^{-}} x$ for some intermediate value $x_{0}=x_{0}\left(S_{t-}, \sigma_{t}, x\right)$, and hence

$$
\xi_{t}(u)=\frac{c^{2}}{\kappa_{t}} F^{\prime}\left(t, S_{t-}, u\right)+\frac{1}{\kappa_{t}} \int_{\mathbb{R}} F^{\prime}\left(t, x_{0}, u\right) x^{2} v_{t}^{\mathbb{Q}}(d x) .
$$

We conclude by (6) that

$$
\sup _{t \in[0, T]}\left|\xi_{t}(u)\right| \leq \sup _{t \in[0, T]}\left(\frac{c_{1}}{\kappa_{t}}\left(c^{2}+\left|\int_{\mathbb{R}} x^{2} v_{t}^{\mathbb{Q}}(d x)\right|\right)\right)<\infty \quad \forall u \quad \mathbb{Q} \text {-a.s. }
$$

This non-random boundedness of $\xi$ implies that $\int_{0}^{t} \xi_{s}(u) d \hat{S}_{s}$ is a square-integrable martingale. Likewise we infer therefore that there exist non-random constants $c_{2}, c_{3}>0$ such that

$$
\begin{gathered}
\sup _{t \in[0, T]}\left|\zeta_{t}^{(1)}(u)\right| \leq c_{2} \sup _{t \in[0, T]} \hat{S}_{t} \forall u \quad \mathbb{Q} \text {-a.s. } \\
\sup _{t \in[0, T]}\left|\zeta_{t}^{(2)}(y, u)\right| \leq c_{3}|y| \sup _{t \in[0, T]} \hat{S}_{t} \forall y, u \quad \mathbb{Q} \text {-a.s. }
\end{gathered}
$$

The $\mathbb{Q}$-square integrability of $\hat{S}_{t}$ together with (8) implies now that $\int_{0}^{t} \zeta_{s}^{(1)}(u) d W_{s}^{\mathbb{Q}}$ is a square-integrable martingale (cf. Protter (2004), Chapter IV, Lemma before Theorem 28). Further with (9) we have that

$$
\begin{aligned}
\mathbb{E}^{\mathbb{Q}}\left[\left(\int_{0}^{T} \int_{\mathbb{R}} \zeta_{s}^{(2)}(y, u) M^{\mathbb{Q}}(d s, d y)\right)^{2}\right] & =\int_{0}^{T} \int_{\mathbb{R}^{\mathbb{Q}}} \mathbb{E}^{\mathbb{Q}}\left[\left(\zeta_{s}^{(2)}(y, u)\right)^{2}\right] v_{s}^{\mathbb{Q}}(d y) d s \\
& \leq T c_{3}^{2} \mathbb{E}^{\mathbb{Q}}\left[\sup _{t \in[0, T]} \hat{S}_{t}^{2}\right] \sup _{s \in[0, T]}\left|\int_{\mathbb{R}} y^{2} v_{s}^{\mathbb{Q}}(d y)\right|<\infty,
\end{aligned}
$$

so $\int_{0}^{t} \int_{\mathbb{R}} \zeta_{s}^{(2)}(y, u) M^{\mathbb{Q}}(d s, d y)$ is also a square-integrable martingale. This finally yields that $K(u)$ is a square-integrable martingale.

Having defined the price processes we introduce now the payment process $\left(Y_{t}\right)_{0 \leq t \leq T}$ which is described by general unit-linked insurance contracts and for which we will derive a hedging strategy. It is given by

$$
Y_{t}=Y_{0}+\sum_{j \in \mathcal{J}} \int_{0}^{t} B_{u}^{-1}\left(I_{u}^{j} G_{u}^{j} d u+\sum_{k: k \neq j} g_{u}^{j k} d N_{u}^{j k}\right), \quad 0 \leq t \leq T .
$$

Here it is important that $S$ and $N^{j k}$ jump simultaneously only with zero probability. Hence the integrals $\int_{0}^{t} B_{u}^{-1} g_{u}^{j k} d N_{u}^{j k}$ are $\mathbb{Q}$-a.s. well-defined. Considering 
the process $Y$ the notion unit-linked becomes finally apparent. The process describes payments that depend on the current state of the policy and on the market value of the stock $S_{t}$. To find now the locally risk-minimizing hedging strategy for the payment process $Y$ (under $\mathbb{P}$ ), we have to derive a risk-minimizing strategy for it under the measure $\mathbb{Q}$ (cf. Appendix A.1). The intrinsic value process $V_{t}^{*}=\mathbb{E}^{\mathbb{Q}}\left[Y_{T} \mid \mathcal{F}_{t}\right], 0 \leq t \leq T$, of $Y$ under $\mathbb{Q}$ and its Galtchouk-KunitaWatanabe decomposition play a crucial role. Like Møller (2001) we define the auxiliary processes $V_{t}^{i}$ for $0 \leq t \leq T$ and $i \in g$ :

$$
V^{i}\left(t, S_{t}\right)=\sum_{j \in \mathcal{J}}\left[\int_{t}^{T} p_{i j}(t, u)\left(F^{j}\left(t, S_{t}, u\right)+\sum_{k: k \neq j} \mu_{u}^{j k} F^{j k}\left(t, S_{t}, u\right)\right) d u\right] .
$$

In Møller (2001) the original measure $\mathbb{P}$ is already a martingale measure for the stock admitting to evaluate the processes $V^{i}$ directly under $\mathbb{P}$. They reflect then the current market value of future benefits less premiums at time $t$ conditional on the policy being in state $i$ at time $t$ and on the value of the stock being $S_{t}$. In this case the processes $V^{i}$ do actually represent the classical state-wise prospective reserve which is the expected value of discounted benefits less premiums under the physical measure $\mathbb{P}$. In our more general setting we have to interpret this under the risk-neutral measure $\mathbb{Q}$ calling $V^{i}$ the state-wise $\mathbb{Q}$-prospective reserve. In conclusion we express the processes $V^{i}$ as

$$
V^{i}(t, s)=\mathbb{E}^{\mathbb{Q}}\left[B_{t}\left(Y_{T}-Y_{t}\right) \mid Z_{t}=i, S_{t}=s\right]
$$

and finally write

$$
V_{t}^{*}=Y_{t}+\sum_{i \in g} I_{t}^{i} B_{t}^{-1} V^{i}\left(t, S_{t}\right) .
$$

Theorem 3.1. The Galtchouk-Kunita-Watanabe decomposition of $V^{*}$ (under $\mathbb{Q}$ ) is given by

$$
V_{t}^{*}=V_{0}^{*}+\int_{0}^{t}\left(\sum_{i \in \mathcal{J}} I_{\tau-}^{i} \xi_{\tau}^{i}\right) d \hat{S}_{\tau}+K_{t}, \quad 0 \leq t \leq T,
$$

where

$$
\xi_{t}^{i}=\sum_{j \in J} \int_{t}^{T}\left(p_{i j}(t, u)\left(\xi_{t}^{j}(u)+\sum_{k: k \neq j} \mu_{u}^{j k} \xi_{t}^{j k}(u)\right)\right) d u
$$

and

$$
\begin{aligned}
K_{t}=\int_{0}^{t}\left(\sum_{i \in \mathcal{J}} I_{\tau-}^{i} \zeta_{\tau}^{(1) i}\right) d W_{\tau}^{\mathbb{Q}} & +\int_{0}^{t} \int_{\mathbb{R}}\left(\sum_{i \in \mathcal{J}} I_{\tau-}^{i} \zeta_{\tau}^{(2) i}(y)\right) M^{\mathbb{Q}}(d \tau, d y) \\
& +\sum_{i, k: i \neq k} \int_{0}^{t} \vartheta_{\tau}^{i k} d M_{\tau}^{i k}
\end{aligned}
$$

is orthogonal to $\hat{S}$ with 


$$
\begin{aligned}
\zeta_{t}^{(1) i} & =\sum_{j \in g} \int_{t}^{T}\left(p_{i j}(t, u)\left(\zeta_{t}^{(1) j}(u)+\sum_{k: k \neq j} \mu_{u}^{j k} \zeta_{t}^{(1) j k}(u)\right)\right) d u, \\
\zeta_{t}^{(2) i}(y) & =\sum_{j \in g} \int_{t}^{T}\left(p_{i j}(t, u)\left(\zeta_{t}^{(2) j}(y, u)+\sum_{k: k \neq j} \mu_{u}^{j k} \zeta_{t}^{(2) j k}(y, u)\right)\right) d u, \\
\vartheta_{t}^{i k} & =B_{t}^{-1}\left(g_{t}^{i k}+V^{k}\left(t, S_{t}\right)-V^{i}\left(t, S_{t}\right)\right) .
\end{aligned}
$$

$\xi_{t}(u), \zeta_{t}^{(1) \cdot}(u)$ and $\zeta_{t}^{(2) \cdot}(y, u)$ are taken from Proposition 3.1.

Proof. The first step is to decompose the process $B_{t}^{-1} V^{i}\left(t, S_{t}\right), i \in \mathcal{J}$. As in Møller (2001) we let

$$
Y_{t}^{i, u}=\sum_{j \in \mathcal{J}} p_{i j}(t, u) B_{t}^{-1}\left(F^{j}\left(t, S_{t}, u\right)+\sum_{k: k \neq j} \mu_{u}^{j k} F^{j k}\left(t, S_{t}, u\right)\right)
$$

for all $i \in \mathcal{I}$ and $0 \leq t \leq u \leq T$ and apply Itô's integration-by-parts formula (cf. Protter (2004), Chapter II, Theorem 22, Corollary 2) yielding

$$
\begin{aligned}
d Y_{t}^{i, u}= & \sum_{j \in g} B_{t}^{-1}\left(F^{j}\left(t, S_{t-}, u\right)+\sum_{k: k \neq j} \mu_{u}^{j k} F^{j k}\left(t, S_{t-}, u\right)\right) d p_{i j}(t, u) \\
& +\sum_{j \in g} p_{i j}(t, u)\left[d\left(B_{t}^{-1} F^{j}\left(t, S_{t}, u\right)\right)+\sum_{k: k \neq j} \mu_{u}^{j k} d\left(B_{t}^{-1} F^{j k}\left(t, S_{t}, u\right)\right)\right] .
\end{aligned}
$$

The quadratic covariation term is identically equal to 0 , since $p_{i j}(\cdot, u)$ is of finite variation and continuous for each $u$. This follows immediately from (4) stating that it is differentiable in the first variable with a bounded derivative on $[0, T]$. Substitution of (4) for $d p_{i j}(t, u)$ yields

$$
\begin{aligned}
d Y_{t}^{i, u}= & \sum_{j \in g} \sum_{k: k \neq j} \mu_{t}^{i k}\left(p_{i j}(t, u)-p_{k j}(t, u)\right) B_{t}^{-1}\left(F^{j}\left(t, S_{t}, u\right)+\sum_{k: k \neq j} \mu_{u}^{j k} F^{j k}\left(t, S_{t}, u\right)\right) d t \\
& +\sum_{j \in g} p_{i j}(t, u)[\cdots] \\
= & \sum_{k: k \neq j} \mu_{t}^{i k}\left(Y_{t}^{i, u}-Y_{t}^{k, u}\right) d t+\sum_{j \in g} p_{i j}(t, u)[\cdots] .
\end{aligned}
$$

Now we apply Proposition 3.1 to the differentials $d\left(B_{t}^{-1} F^{\cdot}\left(t, S_{t}, u\right)\right)$, which yields

$$
\begin{aligned}
d Y_{t}^{i, u} & =\sum_{k: k \neq i} \mu_{t}^{i k}\left(Y_{t}^{i, u}-Y_{t}^{k, u}\right) d t \\
& +\sum_{j \in \mathcal{J}} p_{i j}(t, u)\left[\left(\xi_{t}^{j}(u) d \hat{S}_{t}+\zeta_{t}^{(1) j}(u) d W_{t}^{\mathbb{Q}}+\int_{\mathbb{R}^{\prime}} \zeta_{t}^{(2) j}(y, u) M^{\mathbb{Q}}(d t, d y)\right)\right.
\end{aligned}
$$




$$
\begin{aligned}
& \left.+\sum_{k: k \neq j} \mu_{u}^{j k}\left(\xi_{t}^{j k}(u) d \hat{S}_{t}+\zeta_{t}^{(1) j k}(u) d W_{t}^{\mathbb{Q}}+\int_{\mathbb{R}} \zeta_{t}^{(2) j k}(y, u) M^{\mathbb{Q}}(d t, d y)\right)\right] \\
& =\alpha_{t}^{i, u} d t+\beta_{t}^{i, u} d \hat{S}_{t}+\gamma_{t}^{i, u} d W_{t}^{\mathbb{Q}}+\int_{\mathbb{R}} \delta_{t}^{i, u}(y) M^{\mathbb{Q}}(d t, d y),
\end{aligned}
$$

where

$$
\begin{aligned}
\alpha_{t}^{i, u} & :=\sum_{k: k \neq i} \mu_{t}^{i k}\left(Y_{t}^{i, u}-Y_{t}^{k, u}\right), \\
\beta_{t}^{i, u} & :=\sum_{j \in \mathcal{J}} p_{i j}(t, u)\left(\xi_{t}^{j}(u)+\sum_{k: k \neq j} \mu_{u}^{j k} \xi_{t}^{j k}(u)\right), \\
\gamma_{t}^{i, u} & :=\sum_{j \in \mathcal{J}} p_{i j}(t, u)\left(\zeta_{t}^{(1) j}(u)+\sum_{k: k \neq j} \mu_{u}^{j k} \zeta_{t}^{(1) j k}(u)\right), \\
\delta_{t}^{i, u}(y) & :=\sum_{j \in \mathcal{J}} p_{i j}(t, u)\left(\zeta_{t}^{(2) j}(y, u)+\sum_{k: k \neq j} \mu_{u}^{j k} \zeta_{t}^{(2) j k}(y, u)\right) .
\end{aligned}
$$

This implies

$$
Y_{t}^{i, u}=Y_{0}^{i, u}+\int_{0}^{t} \alpha_{\tau}^{i, u} d \tau+\int_{0}^{t} \beta_{\tau}^{i, u} d \hat{S}_{\tau}+\int_{0}^{t} \gamma_{\tau}^{i, u} d W_{\tau}^{\mathbb{Q}}+\int_{0}^{t} \int_{\mathbb{R}} \delta_{\tau}^{i, u}(y) M^{\mathbb{Q}}(d \tau, d y) .
$$

Note now that $B_{t}^{-1} V^{i}\left(t, S_{t}\right)=\int_{t}^{T} Y_{t}^{i, u} d u$. Hence (cf. Møller (2001)),

$$
\begin{aligned}
B_{t}^{-1} V^{i}\left(t, S_{t}\right)= & \int_{0}^{T} Y_{0}^{i, u} d u+\int_{0}^{T} \int_{0}^{t} 1_{\{\tau \leq u\}} \alpha_{\tau}^{i, u} d \tau d u \\
& +\int_{0}^{T} \int_{0}^{t} 1_{\{\tau \leq u\}} \beta_{\tau}^{i, u} d \hat{S}_{\tau} d u+\int_{0}^{T} \int_{0}^{t} 1_{\{\tau \leq u\}} \gamma_{\tau}^{i, u} d W_{\tau}^{\mathbb{Q}} d u \\
& +\int_{0}^{T} \int_{0}^{t} \int_{\mathbb{R}} 1_{\{\tau \leq u\}} \delta_{\tau}^{i, u}(y) M^{\mathbb{Q}}(d \tau, d y) d u-\int_{0}^{t} Y_{u}^{i, u} d u .
\end{aligned}
$$

Let us evaluate each of the six summands on the right hand sight separately realizing first that

$$
\int_{0}^{T} Y_{0}^{i, u} d u=V^{i}\left(0, S_{0}\right)
$$

Furthermore, as in Møller (2001), it holds that

$$
\begin{aligned}
\int_{0}^{t} Y_{u}^{i, u} d u & =\int_{0}^{t} \sum_{j \in g} \underbrace{p_{i j}(u, u)}_{=1_{\{i=j\}}} B_{u}^{-1}\left(F^{j}\left(u, S_{u}, u\right)+\sum_{k: k \neq j} \mu_{u}^{j k} F^{j k}\left(u, S_{u}, u\right)\right) d u \\
& =\int_{0}^{t} B_{u}^{-1}\left(G_{u}^{i}+\sum_{k: k \neq i} \mu_{u}^{i k} g_{u}^{i k}\right) d u,
\end{aligned}
$$


and

$$
\begin{aligned}
\int_{0}^{T} \int_{0}^{t} 1_{\{\tau \leq u\}} \alpha_{\tau}^{i, u} d \tau d u & =\int_{0}^{t} \int_{\tau}^{T} \alpha_{\tau}^{i, u} d u d \tau \\
& =\int_{0}^{t} \sum_{k: k \neq i} \mu_{\tau}^{i k} \int_{\tau}^{T}\left(Y_{\tau}^{i, u}-Y_{\tau}^{k, u}\right) d u d \tau \\
& =\int_{0}^{t}\left(\sum_{k: k \neq i} \mu_{\tau}^{i k} B_{\tau}^{-1}\left(V^{i}\left(\tau, S_{\tau}\right)-V^{k}\left(\tau, S_{\tau}\right)\right)\right) d \tau
\end{aligned}
$$

The application of Fubini's theorem is allowed here $\mathbb{Q}$-a.s., since the function $(\omega, t, u) \mapsto \alpha_{t}^{i, u}(\omega), i \in \mathcal{J}$, is $O \otimes \mathcal{B}([0, T])$-measurable, and since by (5) we have uniformly for all $t, u$ that $\left|F^{*}\left(t, S_{t}, u\right)\right|<\infty \mathbb{Q}$-a.s. This implies together with the continuity of $\mu_{t}$ that

$$
\int_{0}^{T} \int_{0}^{t} \mathbb{1}_{\{\tau \leq u\}}\left|\alpha_{\tau}^{i, u}\right| d \tau d u<\infty \quad \mathbb{Q} \text {-a.s. }
$$

Additionally, the function $(\omega, t, u) \mapsto \beta_{t}^{i, u}(\omega), i \in \mathcal{J}$, is $\mathcal{P} \otimes \mathcal{B}([0, T])$-measurable and uniformly bounded by a non-random constant (cf. (7)). Fubini's theorem for stochastic integrals (cf. Protter (2004), Chapter IV, Theorem 64) yields then:

$$
\int_{0}^{T} \int_{0}^{t} \mathbb{1}_{\{\tau \leq u\}} \beta_{\tau}^{i, u} d \hat{S}_{\tau} d u=\int_{0}^{t}\left(\int_{\tau}^{T} \beta_{\tau}^{i, u} d u\right) d \hat{S}_{\tau}=\int_{0}^{t} \xi_{\tau}^{i} d \hat{S}_{\tau}
$$

Also $(\omega, t, u) \mapsto \gamma_{t}^{i, u}(\omega), i \in \mathcal{I}$, is $\mathcal{P} \otimes \mathcal{B}([0, T])$-measurable and further there is a non-random constant $\tilde{c}_{2}$ such that

$$
\sup _{t \in[0, T]}\left|\gamma_{\tau}^{i, u}\right| \leq \tilde{c}_{2} \sup _{t \in[0, T]} \hat{S}_{t}, \forall u \quad \mathbb{Q} \text {-a.s., }
$$

since (8) holds and the remaining terms are non-randomly bounded. Hence for all $0 \leq \tau \leq T$ we have that

$$
\left(\int_{\tau}^{T}\left(\gamma_{\tau}^{i, u}\right)^{2} d u\right)^{\frac{1}{2}} \leq \tilde{\tilde{c}}_{2} \sup _{t \in[0, T]} \hat{S}_{t} .
$$

The square-integrability of $\hat{S}$ implies now that the integral of $\left(\int_{s}^{T} \gamma_{s}^{i, u} d u\right)$ with respect to $W^{\mathbb{Q}}$ exists (cf. Protter (2004), Chapter IV, Lemma before Theorem 28). Therefore we may apply the second version of Fubini's Theorem for stochastic integrals (cf. Protter (2004), Chapter IV, Theorem 65) yielding

$$
\int_{0}^{T} \int_{0}^{t} \mathbb{1}_{\{\tau \leq u\}} \gamma_{\tau}^{i, u} d W_{\tau}^{\mathbb{Q}} d u=\int_{0}^{t}\left(\int_{\tau}^{T} \gamma_{\tau}^{i, u} d u\right) d W_{\tau}^{\mathbb{Q}}=\int_{0}^{t} \zeta_{\tau}^{(1) i} d W_{\tau}^{\mathbb{Q}}
$$


Analogously, the function $(\omega, t, u, y) \mapsto \delta_{t}^{i, u}(y), i \in \mathcal{g}$, is $O \otimes \mathcal{B}([0, T]) \otimes \mathcal{B}(\mathbb{R})$ measurable, and by (9) we have that

$$
\sup _{t \in[0, T]}\left|\delta_{t}^{i, u}(y)\right| \leq \tilde{c}_{3}|y| \sup _{t \in[0, T]} \hat{S}_{t}, \quad \forall y, u \quad \mathbb{Q} \text {-a.s., }
$$

for a non-random constant $\tilde{c}_{3}$. This implies for all $0 \leq s \leq T$ that

$$
\left(\int_{s}^{T}\left(\delta_{s}^{i, u}(y)\right)^{2} d u\right)^{\frac{1}{2}} \leq \tilde{\tilde{c}}_{3}|y| \sup _{t \in[0, T]} \hat{S}_{t} .
$$

Hence,

$$
\mathbb{E}^{\mathbb{Q}}\left[\left(\int_{0}^{T} \int_{\mathbb{R}}\left(\int_{s}^{T} \delta_{s}^{i, u}(y) d u\right) M^{\mathbb{Q}}(d s, d y)\right)^{2}\right]<\infty
$$

by the square-integrability of $\hat{S}$ (cf. proof of Proposition 3.1). So we may apply again Fubini's theorem for stochastic integrals:

$\int_{0}^{T} \int_{0}^{t} \int_{\mathbb{R}} \mathbb{1}_{\{s \leq u\}} \delta_{s}^{i, u}(y) M^{\mathbb{Q}}(d s, d y) d u=\int_{0}^{t} \int_{\mathbb{R}}\left(\int_{s}^{T} \delta_{s}^{i, u}(y) d u\right) M^{\mathbb{Q}}(d s, d y) \quad \mathbb{Q}$-a.s.

The computation so far yields the desired decomposition of $B_{t}^{-1} V^{i}\left(t, S_{t}\right)$.

$$
\begin{aligned}
B_{t}^{-1} V^{i}\left(t, S_{t}\right)= & V^{i}\left(0, S_{0}\right)+\int_{0}^{t} \xi_{\tau}^{i} d \hat{S}_{\tau}+\int_{0}^{t} \zeta_{\tau}^{(1) i} d W_{\tau}^{\mathbb{Q}}+\int_{0}^{t} \int_{\mathbb{R}} \zeta_{\tau}^{(2) i}(y) M^{\mathbb{Q}}(d \tau, d y) \\
& -\int_{0}^{t}\left(B_{\tau}^{-1} G_{\tau}^{i}+\sum_{k: k \neq i} \mu_{\tau}^{i k} \vartheta_{\tau}^{i k}\right) d \tau, \mathbb{Q} \text {-a.s. }
\end{aligned}
$$

Using (10) and integration by parts we now obtain the decomposition of $V^{*}$ :

$$
\begin{aligned}
V_{t}^{*} & =Y_{0}+\int_{0}^{t} d Y_{\tau}+\sum_{i \in \mathcal{g}} \int_{0}^{t} d\left(I_{\tau}^{i} B_{\tau}^{-1} V^{i}\left(\tau, S_{\tau}\right)\right) \\
& =Y_{0}+\int_{0}^{t} B_{\tau}^{-1} \sum_{i \in \mathcal{J}}\left(I_{\tau}^{i} G_{\tau}^{i} d \tau+\sum_{k: k \neq i} g_{\tau}^{i k} d N_{\tau}^{i k}\right) \\
& +\sum_{i \in \mathcal{J}}\left(\int_{0}^{t} I_{\tau-}^{i} d\left(B_{\tau}^{-1} V^{i}\left(\tau, S_{\tau}\right)\right)+\int_{0}^{t} B_{\tau}^{-1} V^{i}\left(\tau, S_{\tau-}\right) d I_{\tau}^{i}+\left[I^{i}, B^{-1} V^{i}(\cdot, S .)\right]_{t}\right) .
\end{aligned}
$$

Since $I_{t}^{i}$ is of finite variation, it is a quadratic pure-jump semimartingale (cf. Protter (2004), Chapter II, Theorem 26). So the continuous part of the quadratic covariation above is identically 0 , and we get

$$
\left[I^{i}, B^{-1} V^{i}(\cdot, S)\right]_{t}=I_{0}^{i} B_{0}^{-1} V^{i}\left(0, S_{0}\right)+\sum_{0<\tau \leq t} \Delta I_{\tau}^{i} \Delta\left(B_{\tau}^{-1} V^{i}\left(\tau, S_{\tau}\right)\right) .
$$


This term simplifies considerably because of the initial state of the Markov chain $Z_{t}$ implying $I_{0}^{i}=1_{\{i=0\}}$ and because of the sum of jumps being

$$
\sum_{0<\tau \leq t} \Delta I_{\tau}^{i} \Delta\left(B_{\tau}^{-1} V^{i}\left(\tau, S_{\tau}\right)\right)=0 \quad \mathbb{Q} \text {-a.s., } \quad 0 \leq t \leq T .
$$

This holds, since at least $V^{i}\left(t, S_{t}\right)$ is continuous in probability and the processes $I^{i}$ and $S$ are independent by assumption. Moreover, we only treat càdlàg processes and hence face only countably many jump discontinuities which yields the claim by the $\sigma$-additivity of $\mathbb{Q}$. Additionally observe that

$$
d I_{t}^{i}=\sum_{k: k \neq i}\left(d N_{t}^{k i}-d N_{t}^{i k}\right)
$$

Hence, $\mathbb{Q}$-a.s.,

$$
\begin{aligned}
V_{t}^{*}= & V_{0}^{*}+\sum_{i \in g}\left(\int_{0}^{t} I_{\tau-}^{i} \xi_{\tau}^{i} d \hat{S}_{\tau}+\int_{0}^{t} I_{\tau-}^{i} \zeta_{\tau}^{(1) i} d W_{\tau}^{\mathbb{Q}}+\int_{0}^{t} \int_{\mathbb{R}} I_{\tau-}^{i} \zeta_{\tau}^{(2) i}(y) M^{\mathbb{Q}}(d \tau, d y)\right) \\
& +\sum_{i, k: i \neq k} \int_{0}^{t} B_{\tau}^{-1}\left(V^{k}\left(\tau, S_{\tau-}\right)-V^{i}\left(\tau, S_{\tau-}\right)\right) d N_{\tau}^{i k} \\
& +\int_{0}^{t} B_{\tau}^{-1} \sum_{i \in g}\left(I_{\tau}^{i} G_{\tau}^{i} d \tau+\sum_{k: k \neq i} g_{\tau}^{i k} d N_{\tau}^{i k}\right) \\
& -\sum_{i \in g} \int_{0}^{t} I_{\tau}^{i}\left(B_{\tau}^{-1} G_{\tau}^{i}+\sum_{k: k \neq i} \mu_{\tau}^{i k} \vartheta_{\tau}^{i k}\right) d \tau \\
= & V_{0}^{*}+\int_{0}^{t}\left(\sum_{i \in g} I_{\tau-}^{i} \xi_{\tau}^{i}\right) d \hat{S}_{\tau}+K_{t} .
\end{aligned}
$$

In the last step we used that $d N_{t}^{i k}-I_{t}^{i} \mu_{t}^{i k} d t=d M_{t}^{i k}$. One shows now that this is indeed the desired Galtchouk-Kunita-Watanabe decomposition. Observe therefore that $\left(\sum_{i \in g} I_{t-}^{i} \xi_{t}^{i}\right)$ is $\mathbb{Q}$-a.s. bounded by a non-random constant (cf. proof of Proposition 3.1 and (7)) and so the integral with respect to $\hat{S}_{t}$ is a square-integrable martingale. Also by the boundedness of $\left(\sum_{i \in \mathcal{J}} I_{t-}^{i} \zeta_{t}^{(1) i}\right)$ and of $\left(\sum_{i \in g} I_{t-}^{i} \zeta_{t}^{(2) i}(y)\right)$ (in the sense of (8) and (9), respectively) the integrals with respect to $W^{\mathbb{Q}}$ and $M^{\mathbb{Q}}(\cdot, \cdot)$ are square-integrable martingales. The square-integrability of the $\vartheta^{i k}$, which follows from (5), implies that the integrals with respect to the $M^{i k}$ are also square-integrable martingales (cf. additionally (3)). The orthogonality of $K_{t}$ and $\hat{S}$ is a direct consequence on the one hand of the orthogonal decomposition of $B_{t}^{-1} F^{\cdot}\left(t, S_{t}, u\right)$ and on the other hand of the independence of $S$ and $M^{i k}$.

Corollary 3.1. The unique 0-admissible locally risk minimizing strategy $\varphi$ (under $\mathbb{P}$ ) for the payment process $Y$ of an unit-linked life insurance contract is given by 


$$
\left(\xi_{t}, \eta_{t}\right)=\left(\sum_{i \in \mathcal{J}} I_{t-}^{i} \xi_{t}^{i}, \sum_{i \in \mathcal{J}} I_{t}^{i} B_{t}^{-1} V^{i}\left(t, S_{t}\right)-\sum_{i \in \mathcal{J}} I_{t-}^{i} \xi_{t}^{i} \hat{S}_{t}\right), \quad 0 \leq t \leq T .
$$

The intrinsic risk process (under $\mathbb{Q}$ ) is for $0 \leq t \leq T$ given by

$$
\begin{aligned}
R_{t}(\varphi)= & \sum_{i, j \in \mathcal{g}} I_{t}^{i} \int_{t}^{T}\left(\mathbb{E}^{\mathbb{Q}}\left[\left(\zeta_{\tau}^{(1) j}\right)^{2} \mid \mathcal{F}_{t}\right]+\int_{\mathbb{R}} \mathbb{E}^{\mathbb{Q}}\left[\left(\zeta_{\tau}^{(2) j}\right)^{2} \mid \mathcal{F}_{t}\right] v_{\tau}^{\mathbb{Q}}(d y)\right) p_{i j}(t, \tau) d \tau \\
& +\sum_{i \in \mathcal{g}} I_{t}^{i} \int_{t}^{T} \sum_{j, k: j \neq k} \mathbb{E}^{\mathbb{Q}}\left[\left(\vartheta_{\tau}^{j k}\right)^{2} \mid \mathcal{F}_{t}\right] p_{i j}(t, \tau) \mu_{\tau}^{j k} d \tau,
\end{aligned}
$$

where $\xi^{i}, \zeta^{(1) j}, \zeta^{(2) j}$ and $\vartheta^{j k}$ are taken from Theorem 3.1.

Proof. The hedging strategy is an immediate consequence of Proposition A.1 and Theorem A.2. Further $R_{t}(\varphi)=\mathbb{E}^{\mathbb{Q}}\left[\left(K_{T}-K_{t}\right)^{2} \mid \mathcal{F}_{t}\right]$ with $K_{t}$ as defined in Theorem 3.1. To simplify this expression we use the pairwise orthogonality of the integrals with respect to $W^{\mathbb{Q}}, M^{\mathbb{Q}}(\cdot, \cdot)$ and $M^{i k}$. Note that in our case $\int_{0}^{t} \cdots W_{\tau}^{\mathbb{Q}}$ is a continuous, $\int_{0}^{t} \int_{\mathbb{R}} \cdots M^{\mathbb{Q}}(d \tau, d y)$ is a purely discontinuous martingale and that the $M^{i k}$ are independent of $W^{\mathbb{Q}}$ and $M^{\mathbb{Q}}$ by assumption (for details on the isometries cf. Jacod and Shiryaev (2003), sections I.4.a/b and II.1). For $0 \leq t \leq T$ one gets

$$
\begin{aligned}
\mathbb{E}^{\mathbb{Q}}\left[\left(\int_{t}^{T} \sum_{j \in \mathcal{g}} I_{\tau-}^{j} \zeta_{\tau}^{(1) j} d W_{\tau}^{\mathbb{Q}}\right)^{2} \mid \mathcal{F}_{t}\right] & =\sum_{j \in \mathcal{g}} \int_{t}^{T} \mathbb{E}^{\mathbb{Q}}\left[I_{\tau}^{j} \mid \mathcal{F}_{t}\right] \mathbb{E}^{\mathbb{Q}}\left[\left(\zeta_{\tau}^{(1) j}\right)^{2} \mid \mathcal{F}_{t}\right] d \tau \\
& =\sum_{i, j \in \mathcal{g}} I_{t}^{i} \int_{t}^{T} \mathbb{E}^{\mathbb{Q}}\left[\left(\zeta_{\tau}^{(1) j}\right)^{2} \mid \mathcal{F}_{t}\right] p_{i j}(t, \tau) d \tau
\end{aligned}
$$

Similarly,

$$
\begin{aligned}
& \left.\mathbb{E}^{\mathbb{Q}}\left[\mid \int_{t}^{T} \int_{\mathbb{R}} \sum_{j \in \mathcal{J}} I_{\tau-}^{j} \zeta_{\tau}^{(2) j}(y) M^{\mathbb{Q}}(d \tau, d y)\right)^{2} \mid \mathcal{F}_{t}\right] \\
= & \sum_{i, j \in \mathcal{g}} I_{t}^{i} \int_{t}^{T} \int_{\mathbb{R}} \mathbb{E}^{\mathbb{Q}}\left[\left(\zeta_{\tau}^{(2) j}(y)\right)^{2} \mid \mathcal{F}_{t}\right] p_{i j}(t, \tau) v_{\tau}^{\mathbb{Q}}(d y) d \tau .
\end{aligned}
$$

Finally, (3) and the mutual orthogonality of the $M^{i k}$ itself yields

$$
\begin{aligned}
\mathbb{E}^{\mathbb{Q}}\left[\left(\sum_{j, k: j \neq k} \int_{t}^{T} \vartheta_{\tau}^{j k} d M_{\tau}^{j k}\right)^{2} \mid \mathcal{F}_{t}\right] & =\mathbb{E}^{\mathbb{Q}}\left[\int_{t}^{T} \sum_{j, k: j \neq k}\left(\vartheta_{\tau}^{j k}\right)^{2} \lambda_{\tau}^{j k} d \tau \mid \mathcal{F}_{t}\right] \\
& =\sum_{i \in q} I_{t}^{i} \int_{t}^{T} \sum_{i, r<i+l} \mathbb{E}^{\mathbb{Q}}\left|\left(\vartheta_{\tau}^{j k}\right)^{2}\right| \mathcal{F}_{t} \mid p_{i j}(t, \tau) \mu_{\tau}^{j k} d \tau .
\end{aligned}
$$


The portfolio value of the locally risk-minimizing strategy is $V_{t}(\varphi)=\sum_{i \in g} I_{t}^{i}$ $B_{t}^{-1} V^{i}\left(t, S_{t}\right)$. If we worked under the physical measure $\mathbb{P}, V_{t}(\varphi)$ would exactly correspond to the prospective reserve of a classical life insurance. Thus we call here $V_{t}(\varphi)$ the $\mathbb{Q}$-prospective reserve indicating the evaluation under the measure $\mathbb{Q}$. In the following we comment additionally on the strategy and the hedging risk. The locally risk-minimal investment in the stock at time $t$ depending on the state $i$ of the policy is

$$
\xi_{t}^{i}=\sum_{j \in \mathcal{g}} \int_{t}^{T}\left(p_{i j}(t, u)\left(\xi_{t}^{i}(u)+\sum_{k: k \neq j} \mu_{u}^{j k} \xi_{t}^{j k}(u)\right)\right) d u
$$

where

$$
\xi_{t}(u)=\frac{c^{2}}{\kappa_{t}} F^{\cdot \prime}\left(t, S_{t-}, u\right)+\frac{1}{\sigma_{t} \hat{S}_{t-} \kappa_{t}} \int_{\mathbb{R}} x J \cdot(t, x, u) v_{\tau}^{\mathbb{Q}}(d x) .
$$

The difference from a classical Black-Scholes setting like in Møller (2001) is apparent. If our Lévy process was merely a Brownian motion the measure $v_{t}^{\mathbb{Q}}(d x)$ would be equal to 0 and $\kappa_{t} \equiv c^{2}$ : we would only invest $F^{\cdot{ }^{\prime}}\left(t, S_{t-}, u\right)$, as expected. In the presence of the underlying having jumps the locally risk-minimizing investment in the stock is nearly a weighted sum of $F^{\cdot \prime}\left(t, S_{t-}, u\right)$ and the jump $J^{\cdot}(t, x, u)=B_{t}^{-1}\left\{F^{\cdot}\left(t, S_{t-}+\sigma_{t} S_{t-} x, u\right)-F^{\cdot}\left(t, S_{t-}, u\right)\right\}$. Note that $c^{2} t$ is the variance of the Brownian part $c W_{t}^{\mathbb{Q}}$ and that $\int_{0}^{t} \kappa_{s} d s=c^{2} t+\int_{0}^{t} \int_{\mathbb{R}} x^{2} v_{s}^{\mathbb{Q}}(d x) d s$ is the variance of $L_{t}$ (under $\mathbb{Q}$ ). For the jump part the "weight" is not so obvious but, as seen before, the mean-value theorem yields $J^{\cdot}(t, x, u)=F^{* \prime}\left(t, x_{0}, u\right) \sigma_{t} \hat{S}_{t-} x$ for some suitable intermediate value $x_{0}$ making the weighting more clear. Formula (11) shows that the insurer's intrinsic risk $R_{t}(\varphi)$ of the locally risk-minimizing strategy has two components (dependent on the policy being in state $i$ ):

(a) financial risk driven by trading in the market (only present in an incomplete financial market)

$$
\sum_{j \in \mathcal{J}} \int_{t}^{T}\left(\mathbb{E}^{\mathbb{Q}}\left[\left(\zeta_{\tau}^{(1) j}\right)^{2} \mid \mathcal{F}_{t}\right]+\int_{\mathbb{R}} \mathbb{E}^{\mathbb{Q}}\left[\left(\zeta_{\tau}^{(2) j}(y)\right)^{2} \mid \mathcal{F}_{t}\right] v_{\tau}^{\mathbb{Q}}(d y)\right) p_{i j}(t, \tau) d \tau .
$$

(b) insurance risk driven by mortality

$$
\int_{t}^{T} \sum_{j, k: j \neq k} \mathbb{E}^{\mathbb{Q}}\left[\left(\vartheta_{\tau}^{j k}\right)^{2} \mid \mathcal{F}_{t}\right] p_{i j}(t, \tau) \mu_{\tau}^{j k} d \tau
$$

Note that the pure financial risk appears only in an incomplete financial market and that Møller (2001), considering merely a complete financial market, neglects this significant risk figure. The insurance risk is driven by the sum-atrisk, $\vartheta_{t}^{j k}=B_{t}^{-1}\left(g_{t}^{j k}+V^{k}\left(t, S_{t}\right)-V^{j}\left(t, S_{t}\right)\right)$, and results from the uncertainty of the insured lives. It is equal to the total intrinsic risk in Møller (2001). Each time 
a transition of the policy from state $j$ to state $k$ takes place, the payment $g_{t}^{j k}$ has to be cashed out contributing immediately to the insurer's loss. At the same time the state-wise $\mathbb{Q}$-prospective reserve $V^{j}\left(t, S_{t}\right)$ has to be adjusted to the new state $k$ and is then given by $V^{k}\left(t, S_{t}\right)$. If $V^{k}\left(t, S_{t}\right)-V^{j}\left(t, S_{t}\right)$ is negative, it is used to cover the payment $g_{t}^{j k}$.

Møller (2001) already worked out two interesting examples for a standard Black-Scholes financial market. We have a closer look into them to highlight the differences from a standard Brownian motion setting.

Example 3.2. (Single unit-linked term insurance). We discuss a term insurance issued to a single person against a single premium $P$ at time 0 . The contract specifies the insurance benefit payable immediately upon death of the policy holder if occurred before time $T$. Furthermore we assume that this payment is of unit-linked with guarantee type, that is, the heirs receive a guaranteed deterministic benefit $\gamma e^{\delta t}>0$ for some interest rate $\delta>0$. However, if the value of some reference portfolio $S_{t}$ exceeds this minimal payment they get the amount $S_{t}$. For this contract the state space of the multi-state Markov model is $g=\{0,1\}$, where 0 represents the state policy holder alive and 1 the state policy holder dead. Let the policy holder be of age $x$ at time 0 with remaining lifetime $T_{x}$ after time 0 . The policy holder dying at time $T_{x}$ implies $N_{t}^{01}=1\left(T_{x} \leq t\right)$ and moreover the intensity $\mu^{01}$ of the only possible transition from state 0 to state 1 is the hazard-rate function $\mu$ of $T_{x}$. Note that naturally there are no transitions form state 1 to state 0 . The transition probabilities can be determined by Kolmogorov's backward equations (4). Hence,

$$
p_{00}(t, u)=\exp \left(-\int_{t}^{u} \mu_{\tau} d \tau\right) .
$$

The probability $p_{00}(t, u)$ is the survival probability until time $u$ given the policy holder is alive at time $t$, in actuarial notation this is written as ${ }_{u-t} p_{x+t}$. In contrast $p_{01}(t, u)={ }_{u-t} p_{x+t}=1-p_{00}(t, u)$ is therefore the probability that the person at age $x+t$ dies before time $u$. We have two contract functions different from zero:

$$
g^{01}\left(t, S_{t}\right)=\max \left(S_{t}, \gamma e^{\delta t}\right) \text { and } G_{t}^{0}=\tilde{g}_{t}^{0} 1_{\{t=0\}}=-P 1_{\{t=0\}} .
$$

This means we only consider one price process $F^{01}\left(t, S_{t}, u\right)$ which has to be evaluated depending on the particular Lévy-process model of the financial market. In case the Lévy process is a Brownian motion the Black-Scholes formula can be applied (cf. Møller (2001)). The locally risk-minimizing strategy is given by

$$
\xi_{t}=\mathbb{1}\left(T_{x} \geq t\right) \int_{t}^{T} \xi_{t}^{01}(u) p_{00}(t, u) \mu_{u} d u,
$$

with

$$
\xi_{t}^{01}(u)=\frac{c^{2}}{\kappa_{t}} F^{01,}\left(t, S_{t-}, u\right)+\frac{1}{\sigma_{t} \hat{S}_{t-} \kappa_{t}} \int_{\mathbb{R}} x J^{01}(t, x, u) v_{\tau}^{\mathbb{Q}}(d x)
$$


The locally risk-minimal investment in the bond is $\eta_{t}=1\left(T_{x}>t\right) B_{t}^{-1} V^{0}\left(t, S_{t}\right)-$ $\xi_{t} \hat{S}_{t}$, where

$$
V^{0}\left(t, S_{t}\right)=\int_{t}^{T} F^{01}\left(t, S_{t}, u\right) p_{00}(t, u) \mu_{u} d u
$$

The intrinsic risk is (cf. Corollary 3.1)

$$
\begin{aligned}
R_{t}(\varphi)= & \mathbb{1}\left(T_{x}>t\right) \int_{t}^{T}\left(\mathbb{E}^{\mathbb{Q}}\left[\left(\zeta_{\tau}^{(1) 0}\right)^{2} \mid \mathcal{F}_{t}\right]+\int_{\mathbb{R}} \mathbb{E}^{\mathbb{Q}}\left[\left(\zeta_{\tau}^{(2) 0}(y)\right)^{2} \mid \mathcal{F}_{t}\right] v_{\tau}^{\mathbb{Q}}(d y)\right) p_{00}(t, \tau) d \tau \\
& +\mathbb{1}\left(T_{x}>t\right) \int_{t}^{T} \mathbb{E}^{\mathbb{Q}}\left[\left(\vartheta_{\tau}^{01}\right)^{2} \mid \mathcal{F}_{t}\right] p_{00}(t, \tau) \mu_{\tau}^{01} d \tau,
\end{aligned}
$$

where

$$
\begin{aligned}
\zeta_{t}^{(1) 0} & =\int_{t}^{T} \zeta_{t}^{(1) 01}(u) p_{00}(t, u) \mu_{u} d u, \\
\zeta_{t}^{(2) 0}(y) & =\int_{t}^{T} \zeta_{t}^{(2) 01}(y, u) p_{00}(t, u) \mu_{u} d u .
\end{aligned}
$$

$\zeta_{t}^{(1) 01}(u)$ and $\zeta_{t}^{(2) 01}(y, u)$ are as defined in Proposition 3.1. In this case the sum at risk is given by $\vartheta_{t}^{01}=B_{t}^{-1}\left(g_{t}^{01}-V^{0}\left(t, S_{t}\right)\right)$, which clearly shows the origin of the insurance risk. If the person dies at time $t$ the company's effort is the payment $g_{t}^{01}$ less reserve $V^{0}\left(t, S_{t}\right)$. Further, the initial costs are $C_{0}(\varphi)=V_{0}(\varphi)-P=$ $\int_{0}^{T} p_{00}(0, u) \mu_{u} F^{01}\left(0, S_{0}, u\right) d u-P$. An idea of a minimal premium could therefore be $P=\int_{0}^{T} p_{00}(0, u) \mu_{u} F^{01}\left(0, S_{0}, u\right) d u$.

Example 3.3. (Portfolio of $n$ unit-linked term insurance contracts). Starting form the single unit-linked term insurance with guarantee, we consider now a portfolio of $n$ identical such contracts issued to $n$ policy holders with i.i.d. remaining lifetimes and common hazard-rate function $\mu$. In this case the state space is $g=\{0,1, \ldots, n\}$, where state $j$ corresponds to exactly $j$ policy holders having died. The process $Z$ has transition rates $\lambda_{t}^{j k}=I_{t}^{j} 1_{\{k=j+1\}}(n-j) \mu_{t}$ for $j, k=$ $0, \ldots, n-1$ and the transition probabilities are $p_{j k}(t, u)=0$ for $k<j$ and $p_{j j}(t, u)=$ $\exp \left(-\int_{t}^{u}(n-j) \mu_{\tau} d \tau\right)$. For $k>j$ Kolmogorov's backward equations determine $p_{j k}$. If an insured individual dies, we observe a transition from state $j$ to state $j+1$ and the company has to cash out

$$
g^{j k}\left(t, S_{t}\right)=\mathbb{1}_{\{k=j+1\}} \max \left(S_{t}, \gamma e^{\delta t}\right),
$$

which results in the price process $F^{j k}\left(t, S_{t}, u\right)=1_{\{k=j+1\}} F\left(t, S_{t}, u\right)$. The premium is due at issuing date, thus

$$
G_{t}^{0}=\tilde{g}_{t}^{0} 1_{\{t=0\}}=-n P 1_{\{t=0\}} .
$$


The locally risk-minimizing hedging strategy is (cf. Corollary 3.1)

$$
\begin{aligned}
\xi_{t} & =\sum_{i \in \mathcal{g}} I_{t-}^{i} \xi_{t}^{i}=\int_{t}^{T} \sum_{j=Z_{t-}}^{n} p_{Z_{t-}, j}(t, u)(n-j) \mu_{u} \xi_{t}(u) d u, \\
\eta_{t} & =\sum_{i \in \mathcal{J}} I_{t}^{i} B_{t}^{-1} V^{i}\left(t, S_{t}\right)-\xi_{t} \hat{S}_{t} \\
& =\int_{t}^{T} \sum_{j=Z_{t}}^{n} p_{Z_{t}, j}(t, u)(n-j) \mu_{u} B_{t}^{-1} F\left(t, S_{t}, u\right) d u-\xi_{t} \hat{S}_{t},
\end{aligned}
$$

where $\xi_{t}(u)$ is as in Proposition 3.1. $\left(n-Z_{u}\right)$ given $Z_{t}(u>t)$ follows a binomial distribution with parameters $\left(\left(n-Z_{t}\right), p(t, u)\right)$, where $p(t, u)=\exp \left(-\int_{t}^{u} \mu_{\tau} d \tau\right)(\mathrm{cf}$. Møller (2001)). Hence

$$
\begin{aligned}
& \xi_{t}=\left(n-Z_{t-}\right) \int_{t}^{T} \xi_{t}(u) p(t, u) \mu_{u} d u, \\
& \eta_{t}=\left(n-Z_{t}\right) \int_{t}^{T} B_{t}^{-1} F\left(t, S_{t}, u\right) p(t, u) \mu_{u} d u-\xi_{t} \hat{S}_{t} .
\end{aligned}
$$

We are particularly interested in the intrinsic risk process of this strategy. A laborious computation yields

$$
\begin{aligned}
R_{t}(\varphi)= & \left(n-Z_{t}\right) \int_{t}^{T} p(t, \tau)\left(1-p(t, \tau)+\left(n-Z_{t}\right) p(t, \tau)\right) \rho_{t}(\tau) d \tau \\
& +\left(n-Z_{t}\right) \int_{t}^{T} \mathbb{E}^{\mathbb{Q}}\left[\vartheta_{\tau}^{2} \mid \mathcal{F}_{t}\right] p(t, \tau) \mu_{\tau} d \tau
\end{aligned}
$$

where

$$
\begin{aligned}
\rho_{t}(\tau)= & \mathbb{E}^{\mathbb{Q}}\left[\left(\int_{\tau}^{T} \zeta_{\tau}^{(1)}(u) p(\tau, u) \mu_{u} d u\right)^{2} \mid \mathcal{F}_{t}\right] \\
& +\int_{\mathbb{R}} \mathbb{E}^{\mathbb{Q}}\left[\left(\int_{\tau}^{T} \zeta_{\tau}^{(2)}(y, u) p(\tau, u) \mu_{u} d u\right)^{2} \mid \mathcal{F}_{t}\right] v_{\tau}^{\mathbb{Q}}(d y),
\end{aligned}
$$

and

$$
\vartheta_{\tau}=B_{\tau}^{-1}\left(\max \left(S_{\tau}, \gamma e^{\delta \tau}\right)+V^{j+1}\left(\tau, S_{\tau}\right)-V^{j}\left(\tau, S_{\tau}\right)\right)
$$

$\zeta_{t}^{(1)}(u)$ and $\zeta_{t}^{(2)}(y, u)$ are as in Proposition 3.1. Note that

$$
V^{j+1}\left(\tau, S_{\tau}\right)-V^{j}\left(\tau, S_{\tau}\right)=-\int_{\tau}^{T} F\left(\tau, S_{\tau}, u\right) p(\tau, u) \mu_{u} d u
$$

This has the following interpretation. If an insured person dies at time $\tau$, the insurance company reduces its $\mathbb{Q}$-prospective reserve by the amount $\int_{\tau}^{T} F\left(\tau, S_{\tau}, u\right) p(\tau, u) \mu_{u} d u$ to cover the payment $\max \left(S_{\tau}, \gamma e^{\delta \tau}\right)$. 


\section{A. Appendix}

\section{A.1. General payment stream hedging for semimartingales}

Aligning our setup to Schweizer (1991), we investigate local risk-minimization to be applicable to general payment streams. Throughout the appendix we work on some filtered probability space $\left(\Omega, \mathbb{F},\left(\mathcal{F}_{t}\right)_{0 \leq t \leq T}, \mathbb{P}\right)$ satisfying the usual conditions of right-continuity and completeness. Especially $\mathcal{F}_{0}=\{\emptyset, \Omega\}$ and $\mathcal{F}_{T}=\mathbb{F} . T \in \mathbb{R}$ is fixed and denotes the finite time horizon. Further we consider a financial market with time horizon $T$ consisting of one risky asset (stock) with discounted price process $X$ and a riskless investment alternative (bond) assuming its value to be constant and equal to one. $X=\left(X_{t}\right)_{0 \leq t \leq T}$ is defined to be a semimartingale with decomposition

$$
X=X_{0}+M+A,
$$

such that the assumptions $(X 1)$ to $(X 5)$ of Schweizer (1991) hold.

Definition A.1. A trading strategy $\varphi=(\xi, \eta)$ is a process consisting of the predictable process $\xi=\left(\xi_{t}\right)_{0 \leq t \leq T}$ such that

$$
\mathbb{E}\left[\int_{0}^{T} \xi_{u}^{2} d\langle M\rangle_{u}+\left(\int_{0}^{T}\left|\xi_{u}\right| d|A|_{u}\right)^{2}\right]<\infty,
$$

i.e. $\xi \in \mathrm{L}^{2}\left(\mathbb{P}_{M}\right)$ and $\int_{0}^{T}\left|\xi_{u}\right| d|A|_{u} \in \mathrm{L}^{2}(\mathbb{P})$, and consisting of the adapted process $\eta=\left(\eta_{t}\right)_{0 \leq t \leq T}$ such that the portfolio value $V_{t}(\varphi):=\xi_{t} X_{t}+\eta_{t}$ is càdlàg and satisfies $V_{t}(\varphi) \in \mathrm{L}^{2}(\mathbb{P}), 0 \leq t \leq T$.

Definition A.2. A payment stream or process is an adapted, càdlàg square-integrable process $Y=\left(Y_{t}\right)_{0 \leq t \leq T}$ and the cost process $C(\varphi)$ is defined by

$$
C_{t}(\varphi):=V_{t}(\varphi)-\int_{0}^{t} \xi_{u} d X_{u}+Y_{t}, 0 \leq t \leq T
$$

where $\xi$ satisfies the conditions of Definition A.1.

The payment process $Y$ may be interpreted as a hedger's discounted liabilities towards a buyer of the contract to be hedged, that is, it represents contractual payments. More specifically, from the hedger's point of view, $Y_{t}-Y_{s}, 0 \leq s<$ $t \leq T$, is taken to be the total discounted outgoings less income during the interval $(s, t]$. The introduction of such a process to describe more general payment streams instead of contingent claims (i.e. $H \in \mathrm{L}^{2}, \mathcal{F}_{T}$-adapted and describing a payment at time $T$ ) is due to Møller (2001). This immediately delivers an interpretation of the portfolio value $V_{t}(\varphi)$ : It is the value of the portfolio held at time $t$ after the payments $Y_{t}$ have been made. Since we are primarily concerned 
to hedge the payment process $Y$, it is apparent to concentrate on so called 0 -admissible strategies $\varphi$, i.e. $V_{T}(\varphi)=0 \mathbb{P}$-a.s. As Møller (2001), we additionally consider the following risk process:

$$
R_{t}(\varphi)=\mathbb{E}\left[\left(C_{T}(\varphi)-C_{t}(\varphi)\right)^{2} \mid \mathcal{F}_{t}\right], 0 \leq t \leq T,
$$

given by its càdlàg version. Møller (2001) calls, in analogy to Föllmer and Sondermann (1986), a trading strategy $Y$-mean-self-financing, if its cost process $C_{t}(\varphi)$ is a martingale. For hedging general payment processes in a semimartingale market, we consider now the same optimization problem as in the classical case. The following definition is taken from Schweizer (1991).

Definition A.3. Let $\varphi=(\xi, \eta)$ be a trading strategy and $t \in[0, T]$.

(a) An admissible continuation of $\varphi$ from $t$ on is a trading strategy $\tilde{\varphi}=(\tilde{\xi}, \tilde{\eta})$ satisfying

$\tilde{\xi}_{s}=\xi_{s}$ for $s \leq t, \quad \tilde{\eta}_{s}=\eta_{s}$ for $s<t$,

and

$$
V_{T}(\varphi)=V_{T}(\tilde{\varphi}) \quad \mathbb{P} \text {-a.s. }
$$

(b) $\varphi$ is called risk-minimizing, if for any $t \in[0, T]$ and for any admissible continuation $\tilde{\varphi}$ of $\varphi$ from $t$ on one has

$$
R_{t}(\tilde{\varphi}) \geq R_{t}(\varphi) \quad \mathbb{P} \text {-a.s. }
$$

If $X$ is already a square-integrable $\mathbb{P}$-martingale, the question of finding a riskminimizing trading strategy for general payment processes is completely solved in Møller (2001). In fact it is enough to have a locally square-integrable local martingale $X$. The central idea is to utilize the well-known Galtchouk-KunitaWatanabe decomposition. For completeness we review the result of Møller (2001). We assume for the moment that $X$ is a square-integrable martingale. For a square-integrable process $Y$ one gets for $0 \leq t \leq T$

$$
V_{t}^{*}:=\mathbb{E}\left[Y_{T} \mid \mathcal{F}_{t}\right]=V_{0}^{*}+\int_{0}^{t} \xi_{u}^{Y} d X_{u}+K_{t}^{Y}, \quad \mathbb{P} \text {-a.s. }
$$

where $\xi^{Y} \in \mathrm{L}^{2}\left(\mathbb{P}_{X}\right)$ and $K^{Y}=\left(K_{t}^{Y}\right)_{0 \leq t \leq T}$ is a square-integrable martingale orthogonal to $X$ with $K_{0}^{Y}=0, \mathbb{P}$-a.s. As usual we call two martingales orthogonal if their product is a martingale, or equivalently for square-integrable martingales if their predictable quadratic covariation is identically zero.

Proposition A.1. (Møller). Assume that $X$ is a square-integrable martingale. For every payment process $Y$ there exists an unique 0-admissible risk-minimizing trading strategy $\varphi=(\xi, \eta)$ for $Y$ given by

$$
\left(\xi_{t}, \eta_{t}\right)=\left(\xi_{t}^{Y}, V_{t}^{*}-Y_{t}-\xi_{t}^{Y} X_{t}\right), 0 \leq t \leq T .
$$


The associated risk-process is given by $R_{t}(\varphi)=\mathbb{E}\left[\left(K_{T}^{Y}-K_{t}^{Y}\right)^{2} \mid \mathcal{F}_{t}\right]$.

Proof. Møller (2001).

The difference from the classical theory is that a risk-minimizing strategy $\varphi$ is chosen such that at time $t, 0 \leq t \leq T$, the value of the portfolio is equal to the conditional expected value of the hedger's balance, i.e. $V_{t}(\varphi)=\mathbb{E}\left[Y_{T}-Y_{t} \mid \mathcal{F}_{t}\right]$.

For contingent claim hedging in the semimartingale case things are different. According to Schweizer (1991) it is impossible to find a risk-minimizing hedging strategy that minimizes the process $R_{t}(\varphi)$ uniformly over time and over all admissible continuations of a trading strategy. For a detailed counterexample we refer to Schweizer (2001). Since contingent claim hedging is just a special case $\left(Y_{t}=\mathbb{1}_{\{t \geq T\}} H\right)$ of our more general setting, the projection technique must of course fail here, too. To solve this problem, Schweizer (1991) introduced locally risk-minimizing hedging strategies and showed that they are the right ones to consider in the classical semimartingale case.

\section{Local risk-minimization}

We investigate now if local risk-minimization can be extended from contingent claim hedging to hedging of general payment processes. By intuition this might be quite reasonable, however, in technical terms it is somewhat sophisticated. In particular, the influence of the payment process $Y$ on the concept is not so obvious. Presenting just the main results in this paper we omit to work in detail through the theory of Schweizer (1991) while considering the extended set-up. All this has been worked out and is contained in Riesner (2006b). For a proper definition of the technical term locally risk-minimizing hedging strategy we refer to Schweizer (1991).

Lemma A.1. Assume $X$ satisfies $(X 1)-(X 5)$. Let $Y$ be a payment process and $\varphi=$ $(\xi, \eta)$ a 0 -admissible trading strategy. Then the following statements are equivalent:

(i) $\varphi$ is locally risk-minimizing.

(ii) $\varphi$ is Y-mean-self-financing, and the martingale $C(\varphi)$ is orthogonal to $M$.

Proof. The equivalence of (i) and (ii) follows from Proposition 3.1 and Theorem 3.2 in Schweizer (1990), and Lemma 2.2 in Schweizer (1991).

Remark. The orthogonality results of Schweizer (1991) and Schweizer (1990) suppose the cost process $C_{t}(\varphi)$ to be a square-integrable martingale only. For this reason, adding the square-integrable payment process $Y_{t}$ does not disrupt the classical theory.

One now proceeds to characterize a locally risk-minimizing strategy by the Galtchouk-Kunita-Watanabe decomposition of $C_{T}(\varphi)$ with respect to $\mathbb{P}$ and $M$. 
This yields an optimality equation for the $\xi$ component and the problem of finding a locally risk-minimizing strategy is hence reduced to solve this stochastic optimality equation.

Theorem A.1. Assume that $X$ satisfies $(X 1)-(X 5)$. Let $Y$ be a payment process and let $\varphi=(\xi, \eta)$ be a 0 -admissible trading strategy. Then $\varphi$ is locally risk-minimizing if and only if $\varphi$ is Y-mean-self-financing and $\xi$ satisfies the optimality equation:

$$
\mu^{Y ; \mathbb{P}}-\xi-\mu^{\xi, A ; \mathbb{P}}=0 \quad \mathbb{P}_{M} \text {-a.e. }
$$

Proof. We consider the Galtchouk-Kunita-Watanabe decomposition of $V_{t}^{*}:=$ $\mathbb{E}\left[Y_{T} \mid \mathcal{F}_{t}\right]$, which is for $0 \leq t \leq T$ given by

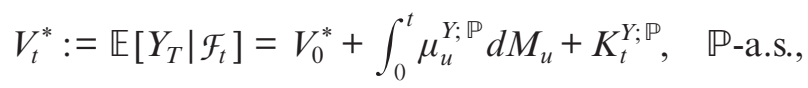

as well as the Galtchouk-Kunita-Watanabe decomposition

$$
\int_{0}^{T} \xi_{u} d A_{u}=\mathbb{E}\left[\int_{0}^{T} \xi_{u} d A_{u}\right]+\int_{0}^{T} \mu_{u}^{\xi, A ; \mathbb{P}} d M_{u}+K_{T}^{\xi, A ; \mathbb{P}}, \mathbb{P} \text {-a.s. }
$$

Note that $\int_{0}^{T} \xi_{u} d A_{u}$ is square-integrable, since $\varphi$ is a trading strategy. The two decompositions together yield

$$
\begin{aligned}
& C_{T}(\varphi)=Y_{T}-\int_{0}^{T} \xi_{u} d X_{u} \\
&=V_{0}^{*}+\int_{0}^{T} \mu_{u}^{Y ; \mathbb{P}} d M_{u}+K_{T}^{Y ; \mathbb{P}}-\int_{0}^{T} \xi_{u} d\left(M_{u}+A_{u}\right) \\
&=\underbrace{V_{0}^{*}-\mathbb{E}\left[\int_{0}^{T} \xi_{u} d A_{u}\right]}_{=C_{0}(\varphi)}+\int_{0}^{T}\left(\mu_{u}^{Y ; \mathbb{P}}-\xi_{u}-\mu_{u}^{\xi, A ; \mathbb{P}}\right) d M_{u}+K_{T}^{Y ; \mathbb{P}}-K_{T}^{\xi ;, A ; \mathbb{P}}, \\
& \mathbb{P} \text {-a.s. }
\end{aligned}
$$

Lemma A.1 yields then the result.

\section{Finding a locally risk-minimizing strategy}

In this section it is shown that an unique solution to the optimality equation exists. First one adds some more structure on the considered probability space (cf. Schweizer (1991).

Definition A.4. Two stochastic processes $M$ and $N$ are said to form a $\mathbb{P}$-basis of $\mathrm{L}^{2}(\mathbb{P})$ if both $M$ and $N$ are square-integrable martingales under $\mathbb{P}$ and $M-M_{0}$ and $N-N_{0}$ are $\mathbb{P}$-orthogonal (as martingales). Further, if every $H \in \mathrm{L}^{2}(\mathbb{P})$ has a unique representation 


$$
H=\mathbb{E}[H]+\int_{0}^{T} \mu_{u}^{H ; \mathbb{P}} d M_{u}+\int_{0}^{T} v_{u}^{H ; \mathbb{P}} d N_{u} \mathbb{P} \text {-a.s. }
$$

for two predictable processes $\mu^{H ; \mathbb{P}} \in \mathrm{L}^{2}\left(\mathbb{P}_{M}\right)$ and $v^{H ; \mathbb{P}} \in \mathrm{L}^{2}\left(\mathbb{P}_{N}\right)$.

The following assumptions are added to the model.

$(P 1)$ There exists a process $N=\left(N_{t}\right)_{0 \leq t \leq T}$ such that $M$ and $N$ form a $\mathbb{P}$-basis of $\mathrm{L}^{2}(\mathbb{P})$.

$(P 2)$ There exists a probability measure $\mathbb{Q}$ equivalent to $\mathbb{P}$ such that $X$ and $N$ form a $\mathbb{Q}$-basis of $L^{2}(\mathbb{Q})$.

Because of $(P 2)$ the considered financial market must be free of arbitrage. Schweizer (1991) provides moreover a formula for the Radon-Nikodým density of the measure $\mathbb{Q}$, called the Föllmer-Schweizer measure, and shows that it is essentially unique. This formula provides an insight into the performed Girsanov change of measure removing the drift $A$ from $X$. It gives furthermore an idea of how to construct the measure $\mathbb{Q}$.

Consider now a payment process $Y=\left(Y_{t}\right)_{0 \leq t \leq T}$ which is also square-integrable with respect to $\mathbb{Q}$. Under assumption $(P 2)$ we apply Proposition A.1 to the hedging problem and find an unique hedging strategy $\varphi^{Y ; \mathbb{Q}}=\left(\xi^{Y ; \mathbb{Q}}, \eta^{Y ; \mathbb{Q}}\right)$ which is risk-minimizing with respect to $\mathbb{Q}$ and satisfies $V_{T}\left(\varphi^{Y ; \mathbb{Q}}\right)=0$. $\xi^{Y ; \mathbb{Q}}$ is given by the Kunita-Watanabe decomposition

$$
Y_{T}=\mathbb{E}^{\mathbb{Q}}\left[Y_{T}\right]+\int_{0}^{T} \xi_{u}^{Y ; \mathbb{Q}} d X_{u}+\int_{0}^{T} v_{u}^{Y ; \mathbb{Q}} d N_{u} \mathbb{Q} \text {-a.s. }
$$

and

$$
\eta_{t}^{Y ; \mathbb{Q}}=\mathbb{E}^{\mathbb{Q}}\left[Y_{T} \mid \mathcal{F}_{t}\right]-Y_{t}-\xi_{t}^{Y ; \mathbb{Q}} X_{t}, \quad \mathbb{Q} \text {-a.s., } \quad 0 \leq t \leq T .
$$

The idea is now to use $\varphi^{Y ; \mathbb{Q}}$ as candidate for a locally risk-minimizing strategy under $\mathbb{P}$.

Theorem A.2. Assume that $X$ satisfies $(X 1)-(X 5)$ and that $(P 1)$ and $(P 2)$ hold. Let $Y$ be a payment process and square-integrable with respect to $\mathbb{Q}$. Suppose additionally that $v^{Y ; \mathbb{Q}} \in \mathrm{L}^{2}\left(\mathbb{P}_{N}\right)$ and that $\xi^{Y ; \mathbb{Q}}$ satisfies (11). Then $\varphi^{Y ; \mathbb{Q}}$ is locally risk-minimizing with respect to $\mathbb{P}$.

Proof. According to Theorem A.1 first one has to show that $\xi^{Y ; \mathbb{Q}}$ is a solution to the optimality equation (13). This is proved as in the first part of Theorem 3.2 in Schweizer (1991) where $\xi^{H ; \mathbb{\varpi}}$ has to be replaced by $\xi^{Y ; \mathbb{Q}}$ and $H$ by $Y_{T}$. It remains now to show that $\varphi^{Y ; \mathbb{Q}}$ is $Y$-mean-self-financing with respect to $\mathbb{P}$. (14) yields

$$
C_{T}\left(\varphi^{Y ; \mathbb{Q}}\right)=\underbrace{V_{T}\left(\varphi^{Y ; \mathbb{Q}}\right)}_{=0}-\int_{0}^{T} \xi_{u}^{Y ; \mathbb{Q}} d X_{u}+Y_{T}=\mathbb{E}^{\mathbb{Q}}\left[Y_{T}\right]+\int_{0}^{T} v_{u}^{Y, \mathbb{Q}} d N_{u} .
$$


Further,

$$
\begin{aligned}
C_{t}\left(\varphi^{Y ; \mathbb{Q}}\right) & =V_{t}\left(\varphi^{Y ; \mathbb{Q}}\right)-\int_{0}^{t} \xi_{u}^{Y ; \mathbb{Q}} d X_{u}+Y_{t} \\
& =\xi_{t}^{Y ; \mathbb{Q}} X_{t}+\eta_{t}^{Y ; \mathbb{Q}}-\int_{0}^{t} \xi_{u}^{Y ; \mathbb{Q}} d X_{u}+Y_{t} .
\end{aligned}
$$

Recall that $\eta_{t}^{Y ; \mathbb{Q}}=\mathbb{E}^{\mathbb{Q}}\left[Y_{T} \mid \mathcal{F}_{t}\right]-Y_{t}-\xi_{t}^{Y ; \mathbb{Q}} X_{t}$. Using $(P 2)$ we get

$$
\mathbb{E}^{\mathbb{Q}}\left[Y_{T} \mid \mathcal{F}_{t}\right]=\mathbb{E}^{\mathbb{Q}}\left[Y_{T}\right]+\int_{0}^{t} \xi_{u}^{Y ; \mathbb{Q}} d X_{u}+\int_{0}^{t} v_{u}^{Y ; \mathbb{Q}} d N_{u}
$$

Hence, $C_{t}\left(\varphi^{Y ; \mathbb{Q}}\right)=\mathbb{E}^{\mathbb{Q}}\left[Y_{T}\right]+\int_{0}^{t} v_{u}^{Y ; \mathbb{Q}} d N_{u}, 0 \leq t \leq T$. The claim follows, since $N$ is a martingale under $\mathbb{P}$ and $v^{Y ; \mathbb{Q}} \in \mathrm{L}^{2}\left(\mathbb{P}_{N}\right)$.

Summarizing the Appendix we remark that the local risk-minimization theory for the classical case transfers to the case of general payment processes and is applied in the same manner. First, one removes the drift of the semimartingale $X$ performing an equivalent change of measure to the Föllmer-Schweizer measure $\mathbb{Q}$ and second, one applies the theory of Møller (2001) to find a risk-minimizing hedging strategy under $\mathbb{Q}$. This strategy is then locally risk-minimizing with respect to the original measure $\mathbb{P}$.

\section{ACKNOWLEDGEMENT}

I very much appreciate the valuable discussions with my supervisors Ulrich Stadtmüller and Rüdiger Kiesel. Special thanks go to Nick Bingham for his very helpful "red-ink" job and to an unknown referee for suggestions improving the presentation of the paper.

\section{REFERENCES}

Aase, K. and Persson, S.A. (1994) Pricing of unit-linked life insurance policies. Scandinavian Actuarial Journal 1, 26-52.

Chan, T. (1999) Pricing contingent claims on stocks driven by Lévy processes. The Annals of Applied Probability 9(2), 504-528.

Föllmer, H. and SondermanN, D. (1986) Hedging of non-redundant contingent claims. Contributions to Mathematical Economics. Hildenbrand, W., Mas-Colell, A. (eds.), North-Holland, 205-223.

HoEM, J. (1969) Markov chain models in life insurance. Blätter der Deutschen Gesellschaft für Versicherungsmathematik 9, 91-107.

Jacod, J. and Shiryaev, A.N. (2003) Limit Theorems for Stochastic Processes. 2nd ed., Springer, Berlin-Heidelberg-New York.

Kallenberg, O. (2002) Foundations of Modern Probability. 2nd ed., Springer, New York-BerlinHeidelberg.

MøLleR, T. (1998) Risk-minimizing hedging strategies for unit-linked life insurance contracts. ASTIN Bulletin 28, 1-47. 
Møller, T. (2001) Risk-mimizing hedging strategies for insurance payment processes. Finance and Stochastics 5(4), 419-446.

Protter, P. (2004) Stochastic Integration and Differential Equations. 2nd ed., Springer, Berlin-Heidelberg-New York.

RieSNER, M. (2006a) Hedging life insurance contracts in a Lévy process financial market. Insurance: Mathematics and Economics 38(3), 599-608.

RIESNER, M. (2006b) Unit-linked life insurance in Lévy-process financial markets. Dissertation, Ulm University.

SCHWEIzer, M. (1990) Risk-minimality and orthogonality of martingales. Stochastics and Stochastic Reports 30, 123-131.

Schweizer, M. (1991) Option hedging for semimartingales. Stochastic Processes and their Applications 37, 339-363.

Schweizer, M. (2001) A guided tour through quadratic hedging approaches. Handbooks in Mathematical Finance: Option Pricing, Interest Rates and Risk Management. Jouini, E., Cvitanic, J., Musiela, M. (eds.), Cambridge University Press, Cambridge, 538-574.

MARTIN RIESNER

Abteilung für Zahlentheorie und Wahrscheinlichkeitstheorie

Fakultät für Mathematik und Wirtschaftswissenschaften

Universität Ulm

Helmholtzstrasse 18

89069 Ulm

Germany

Tel.: +497315023514

Fax: +497315023516

E-mail:martin.riesner@alumni.uni-ulm.de 